\title{
Atypical Hemolytic Uremic Syndrome after ChAdOx1 nCoV-19 Vaccination in a Patient with Homozygous CFHR3/CFHR1 Gene Deletion
}

\author{
Francisco Ferrer Marisa Roldão Cátia Figueiredo Karina Lopes \\ Nephrology Department, Centro Hospitalar do Médio Tejo, Torres Novas, Portugal
}

\section{Keywords}

Thrombotic microangiopathy · Atypical hemolytic uremic syndrome $\cdot$ Severe acute respiratory syndrome coronavirus $2 \cdot$ Vaccine

\begin{abstract}
Hemolytic uremic syndrome (HUS) is a thrombotic microangiopathy (TMA) affecting the kidneys. Compared with typical HUS due to an infection from shiga toxin-producing Escherichia coli, atypical HUS involves a genetic or acquired dysregulation of the complement alternative pathway. In the presence of a mutation in a complement gene, a second trigger is often necessary for the development of the disease. We report a case of a 54-year-old female, with a past medical history of pulmonary tuberculosis, who was admitted to the emergency service with general malaise and reduction in urine output, 5 days after vaccination with ChAdOx $1 \mathrm{nCoV}$ 19. Laboratory results revealed microangiopathic hemolytic anemia, thrombocytopenia, and acute kidney injury. Given the clinical picture of TMA, plasma exchange (PEX) was immediately started, along with hemodialysis. Complementary laboratory workup for TMA excluded thrombotic thrombocytopenic purpura and secondary causes. Complement
\end{abstract}

karger@karger.com www.karger.com/nef

(C) 2021 S. Karger AG, Basel

Karger $\stackrel{2}{=}$ study revealed normal levels of factors $\mathrm{H}, \mathrm{B}$, and I, normal activity of the alternate pathway, and absence of anti-factor $\mathrm{H}$ antibodies. Genetic study of complement did not show pathogenic variants in the 12 genes analyzed, but revealed a deletion in gene CFHR3/CFHR1 in homozygosity. Our patient completed 10 sessions of PEX, followed by eculizumab, with both clinical and laboratorial improvement. Actually, given the short time lapse between vaccination with ChAdOx1 nCoV-19 and the clinical manifestations, we believe that vaccine was the trigger for the presentation of aHUS in this particular case.

(c) 2021 S. Karger AG, Basel

\section{Introduction}

Thrombotic microangiopathies (TMA) are rare disorders, with varied etiologies, characterized by the defining features of microangiopathic hemolytic anemia (Coombs negative) and thrombocytopenia. These disorders are associated with significant morbidity and mortality, related mainly to endothelial damage and thrombosis of the affected organs (with subsequent injury). Hemolytic uremic syndrome (HUS) is a TMA where the pathological 
findings of vascular endothelial injury are observed mainly in the kidney, and acute kidney injury (AKI) is a prominent feature [1].

HUS classification can be challenging because a variety of physiopathologic mechanisms lead to the same histologic, clinical, and laboratorial manifestations [2]. Different from "typical" HUS due to shiga toxin-producing Escherichia coli or from HUS associated/precipitated by autoimmune diseases, malignancies, or even medications, atypical HUS (aHUS) is mainly related to complement pathway disorders (either acquired or genetic) [1, 3 , 4]. However, in about $30 \%$ cases of aHUS, the mechanisms for disease are unknown [1]. As a rare disease, HUS diagnosis is not always straightforward and requires an index of clinical suspicion for the early initiation of treatment, to minimize permanent organ damage.

The evolving knowledge of complement cascade has been determinant in elucidating the physiopathology of aHUS and in the advent of new and targeted therapies of the disorder. However, for a complement-mediated aHUS to manifest, in addition to a mutation in a complement gene and an "at-risk" haplotype, a second trigger is often necessary [5]. This second trigger is often related to infections, pregnancies, or other intercurrent illnesses. We describe a case of aHUS in a middle-aged woman, 5 days after being vaccinated with ChAdOx1 nCoV-19, a chimpanzee adenovirus-vectored vaccine encoding the spike protein antigen of severe acute respiratory syndrome coronavirus 2 (SARS-CoV-2).

\section{Case Report}

The authors present a case of a 54-year-old Caucasian female, with a past medical history relevant for pulmonary tuberculosis (diagnosed in December 2014, treated with 4 tuberculostatics isoniazid, rifampicin, ethambutol, and pyrazinamide - during 9 months and in complete remission since September 2015, but with pulmonary sequelae) and a obstetrical history of 3 pregnancies and 1 spontaneous abortion (during the first trimester, with no clear cause). Due to her antecedents of pulmonary disease, this patient was vaccinated with ChAdOx1 nCoV-19 (Batch No. ABV3025) at the beginning of March 2021. Soon after vaccine administration, she complained of "flu-like" symptoms that resolved with paracetamol. Five days later, she was admitted to our emergency department with general malaise, abdominal pain, myalgia, vomits, and low urine output; there were no complaints of respiratory symptoms or diarrhea. Physical examination revealed no significant abnormalities, apart from arterial hypertension $(170 / 110 \mathrm{~mm}$ $\mathrm{Hg}$ ). Initial laboratory workup was relevant for de novo thrombocytopenia $(50,000 / \mathrm{uL})$, anemia $(10.3 \mathrm{~g} / \mathrm{dL})$ - with schistocytes on peripheral blood smear, renal dysfunction (creatinine $5 \mathrm{mg} / \mathrm{dL}$ and urea $96 \mathrm{mg} / \mathrm{dL}$ ), elevated C-reactive protein $(17 \mathrm{mg} / \mathrm{dL})$, lactate dehydrogenase (LDH) $(4,954 \mathrm{IU} / \mathrm{L}$ - normal range <248 IU/L), undetectable haptoglobin levels $(<5.83 \mathrm{mg} / \mathrm{dL})$, and normal direct and indirect serum bilirubin. Coagulation tests (prothrombin time and activated partial thromboplastin time) were also normal. Urinalysis showed hemoglobinuria $(1 \mathrm{mg} / \mathrm{dL})$, proteinuria $(100 \mathrm{mg} /$ $\mathrm{dL})$, leukocyturia (10/hpf), and hematuria (50/hpf). Renal ultrasound had revealed normal kidneys without echographic signs of obstruction.

Given the clinical picture of AKI, with severe thrombocytopenia, anemia, elevated LDH, undetectable haptoglobin, and negative Coombs test (suggesting microangiopathic hemolytic anemia), a TMA was suspected as the underlying disorder, and a complete laboratory workup was performed, consisting of serum complement levels (including $\mathrm{CH} 50$ and AH50), immunologic study, ADAMTS13 activity, antibody anti-ADAMTS13, factors $\mathrm{H}$, $\mathrm{B}$, and I, antibody anti-factor $\mathrm{H}$, stool culture for shiga toxin-producing microbes, and genetic testing for mutations in complement regulatory or effector genes. Plasma exchange (PEX) by the filtration method was immediately started, with 1.5 plasma volumes of fresh frozen plasma. During the first PEX session, after the removal of $2.7 \mathrm{~L}$ of plasma, the patient presented with an urticariform reaction (pruriginous rash, involving mainly arms and legs, with wheezing), attributed to plasma protein allergy and promptly treated by the suspension of the session, intravenous hydrocortisone $(100 \mathrm{mg})$, and clemastine $(2 \mathrm{mg})$. The PEX sessions were resumed on the following day, with the administration of clemastine and hydrocortisone at the beginning, and no other allergic adverse events were described. She accomplished 10 sessions of PEX (5 consecutive and 5 on alternate days), with normalization of platelet count and anemia improvement; LDH also returned to normal range levels. Considering anuric AKI and clinical signs of volume overload, hemodialysis was started after the second session of PEX.

Further laboratory workup (Table 1) concerning TMA was relevant for low C3 levels, normal activity of ADAMTS13, absence of anti-ADAMTS13 antibodies, absence of detection of shiga toxinproducing E. coli in stool culture (including O157 strain), normal levels of factors $\mathrm{H}, \mathrm{B}$, and I, a normal activity of the alternate complement pathway (as depicted by normal levels of AH50), and absence of anti-factor $\mathrm{H}$ antibodies. Molecular genetic study of 12 genes of the next-generation sequencing (NGS) panel (CFH, CD46 [MCP], CFI, C3, THBD, CFB, CFHR5, CFHR1, CFHR3, CFHR4, DGKE, and ADAMTS13), after extraction of genomic DNA using QIAsymphony SP (Qiagen), followed by sequencing with the mentioned panel and analysis with Ion ReporterTM software, did not recognize any pathogenic variants in the analyzed genes, but it identified a polymorphism described as a possible risk factor for aHUS development: deletion in homozygosity of CFHR3/CFHR1 (see below).

Given the absence of a secondary cause for TMA, a diagnosis of aHUS was made, and therapy with eculizumab $900 \mathrm{mg} /$ week was started (after vaccination with antimeningococcal B and conjugated tetravalent meningococcal vaccines), with concomitant prophylaxis for meningococcal invasive infection with amoxicillin $500 \mathrm{mg}$ twice daily. After the first administration of eculizumab, the patient resumed diuresis, renal function partially recovered, and hemodialysis was suspended after 10 treatments. No other PEX sessions were needed, and the patient was discharged with a hemoglobin level of $11.6 \mathrm{~g} / \mathrm{dL}$, normal platelet count, normal $\mathrm{LDH}$, creatinine $4.4 \mathrm{~g} / \mathrm{dL}$, and urea $114 \mathrm{mg} / \mathrm{dL}$ - after 2 administrations of eculizumab 900/week (Fig. 1). At the time we wrote this manuscript, our patient has completed 4 weekly administrations 
Table 1. Immunological, autoimmune, and complement study

\begin{tabular}{lll}
\hline Analytical parameters & Value & Reference value \\
\hline C3 & $70.70 \mathrm{mg} / \mathrm{dL}$ & $79.00-152.00$ \\
C4 & $21.90 \mathrm{mg} / \mathrm{dL}$ & $16.00-38.00$ \\
CH50 & $92 \mathrm{U} / \mathrm{mL}$ & $>42$ \\
AH50, $\%$ & $>137$ & $>70$ \\
Anti-cardiolipin antibody (ELISA) & IgM: $1.90 \mathrm{MPL} / \mathrm{mL}$ & Positive: $>40.0$ \\
& & Weakly positive: $10.0-40.0$ \\
& & Negative: $<10.0$ \\
Antinuclear antibody (ELISA) & IgG: $1.10 \mathrm{GPL} / \mathrm{mL}$ & \\
& $0.10(\mathrm{ratio})$ & Positive: $>1.1$ \\
pANCA (indirect immunofluorescence) & & Doubtful: $0.9-1.1$ \\
CANCA (indirect immunofluorescence) & Negative: $<0.9$ \\
Anti-glomerular basement membrane antibody & Negative & - \\
(immunofluorescence) & $0.90 \mathrm{U} / \mathrm{mL}$ & - \\
& & Positive: $>10.0$ \\
Anti-dsDNA antibody (ELISA) & & Doubtful: $7.0-10.0$ \\
& & Negative: $<7.0$ \\
Complement factor I & $<10.00 \mathrm{IU} / \mathrm{mL}$ & Negative: $<100$ \\
Complement factor B & & Positive: $\geq 100$ \\
Complement factor H & $2.69 \mathrm{mg} / \mathrm{dL}$ & $2.38-3.18$ \\
Complement factor H antibody (ELISA) & $38.50 \mathrm{mg} / \mathrm{dL}$ & $17.04-51.06$ \\
ADAMTS13 activity & $54.80 \mathrm{mg} / \mathrm{dL}$ & $47.45-65.99$ \\
ADAMTS13 antibody (ELISA) & $8.05 \mathrm{UA} / \mathrm{mL}$ & $<27$ \\
\hline & $0.79 \mathrm{UI} / \mathrm{mL}$ & $\geq 0.67$ \\
& $1.19 \mathrm{U} / \mathrm{mL}$ & $<15$ \\
\hline
\end{tabular}

of eculizumab (900 mg/each), followed by 2 administrations 15 days apart (1,200 mg/each), with no evidence of hemolysis (hemoglobin of $13 \mathrm{~g} / \mathrm{dL}$ and normal $\mathrm{LDH}$ ), normal platelet count, and improvement of renal function (serum creatinine of $2 \mathrm{mg} / \mathrm{dL}$ ).

\section{Discussion}

Since the beginning of vaccination for SARS-CoV-2, millions of vaccines have been administered around the world. While the reported adverse events of vaccination are scarce, the magnitude of some dents public opinion and makes the worldwide campaign to fight the pandemic difficult. These adverse events also contribute to many governments' erratic strategy in the administration of vaccines.

Two reports of serious adverse events related to the administration of ChAdOxl $\mathrm{nCoV}$ vaccine in Austria, Germany, and Norway, were recently published $[6,7]$. These serious reactions to this vaccine consisted of thromboembolic episodes, mainly in young women, associated with thrombocytopenia and the production of PF4-heparin antibodies, similar to what happens in heparin-induced thrombocytopenia - a condition called by the authors as vaccine-induced immune thrombotic thrombo- cytopenia. In these conditions, the authors did not report the existence of hemolysis, and there was also a good response to platelet transfusion.

In our patient, the evidence of hemolysis was clear since hospital admission; indeed, the association of nonimmune hemolytic anemia and thrombocytopenia with acute renal dysfunction made us think of a TMA. After excluding other causes of TMA, a diagnosis of aHUS was made straightforward. Genetic study did not identify any pathogenic variants in the 12 genes analyzed; the only finding was the deletion of CFHR3/CFHR1 in homozygosity. This deletion appears as a polymorphism in normal population, with a frequency of $2 \%$ in European countries [8]; however, when present in homozygosity, this polymorphism is associated with aHUS and with production of anti-factor $\mathrm{H}$ antibodies (absent in our patient), in the presence of a trigger $[8,9]$. The absence of pathogenic variants in genes encoding complement-related proteins, despite the presence of the mentioned polymorphism (but without anti-factor $\mathrm{H}$ antibodies), impedes us from identifying a clear genetic predisposition to aHUS in this patient. However, as the only event close to disease presentation was the administration of ChAdOx1 $\mathrm{nCoV}$ vaccine, we think this could have been the trigger 


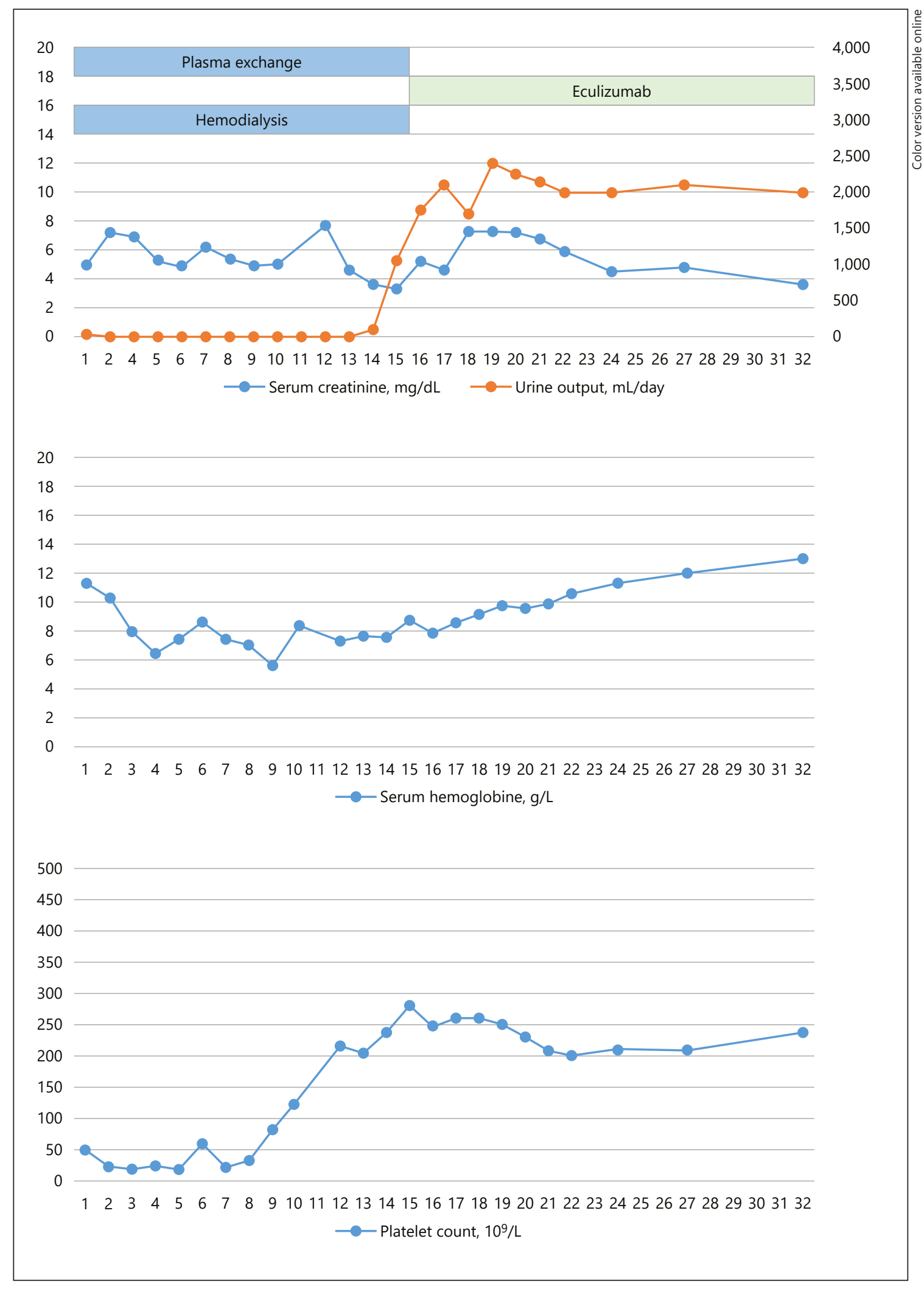

Fig. 1. Evolution of serum creatinine, urine output, serum hemoglobin, and platelet count in the first 31 days after diagnosis. 
event to reveal the alternate pathway complement disorder. So far for us is not possible to assume that the adenovirus vector with spike glycoprotein itself or one of the excipients of the vaccine is the underlying culprit for development of aHUS. Indeed, this patient had already been vaccinated with several vaccines (against influenza, tetanus, pneumococcus, and meningococcus - the latter as prophylaxis before eculizumab administration) with no significant collateral side effects reported. The good response to terminal complement blockade after the first 4 weekly doses made us planning further administration of eculizumab 1,200 mg every 2 weeks. At this time, we do not know the optimal duration of targeted therapy, but we think that in accordance with consensus opinions, it should be maintained for at least 6-12 months, with a very close follow-up after discontinuation [3].

To the best of our knowledge, this is the first report of HUS associated with COVID-19 vaccination, namely, with a chimpanzee adenovirus-vectored vaccine. Considering worldwide mass vaccination, further observations should be reported to confirm this risk, with vaccination acting as a trigger for abnormal complement activation. However, despite the importance of these singular cases to the medical community, we should never forget that given the inexistence of an effective treatment of SARS$\mathrm{CoV}-2$, the only pathway to eliminate the pandemic is mass worldwide vaccination.

\section{Statement of Ethics}

This is a case report. The Ethics Committee of Centro Hospitalar do Médio Tejo has confirmed that no ethical approval is required. Informed consent was obtained from the patient reported in this case. The participant has consented to the submission of the case report to the journal.

\section{Conflict of Interest Statement}

The authors have no conflicts of interest to declare that are relevant to the content of this article.

\section{Funding Sources}

No funds, grants, or other support was received.

\section{Author Contributions}

F.F., M.R., and C.F. contributed equally to this work, by following the patient and by writing the manuscript. K.L. critically revised the manuscript.

\section{Data Availability Statement}

Data and material and code availability is not applicable.

\section{Acknowledgments}

The authors thank Teresa Fidalgo PharmD, PhD, for assistance in the interpretation of genetic results and Fernando Carrera, MD, for assistance with language editing.

\section{References}

1 Fakhouri F, Zuber J, Frémeaux-Bacchi V, Loirat C. Haemolytic uraemic syndrome. Lancet. 2017 Aug 12;390(10095):681-96.

2 Loirat C, Fakhouri F, Ariceta G, Besbas N, Bitzan M, Bjerre A, et al. An international consensus approach to the management of atypical hemolytic uremic syndrome in children. Pediatr Nephrol. 2016 Jan;31(1):15-39.

3 Azevedo A, Faria B, Teixeira C, Carvalho F, Neto G, Santos J, et al. Portuguese consensus document statement in diagnostic and management of atypical hemolytic uraemic syndrome. Port J Nephrol Hypert. 2018 Sep; 32(39):211-32.
4 Noris M, Caprioli J, Bresin E, Mossali C, Pianetti G, Gamba S, et al. Relative role of genetic complement abnormalities in sporadic and familial aHUS and their impact on clinical phenotype. Clin J Am Soc Nephrol. 2010 Oct;5(10):1844-59.

5 Noris M, Remuzzi G. Atypical hemolytic-uremic syndrome. N Engl J Med. 2009 Oct 22; 361(17):1676-87.

6 Greinacher A, Thiele T, Warkentin TE, Weisser K, Kyrle PA, Eichinger S. Thrombotic thrombocytopenia after ChAdOxl nCov19 vaccination. N Engl J Med. 2021 Jun 3; 384(22):2092-101.
7 Schultz NH, Sørvoll IH, Michelsen AE Munthe LA, Lund-Johansen F, Ahlen MT, et al. Thrombosis and thrombocytopenia after ChAdOx1 nCoV-19 vaccination. N Engl J Med. 2021 Jun 3;384(22):2124-30.

8 Skerka C, Chen Q, Fremeaux-Bacchi V, Roumenina LT. Complement factor $\mathrm{H}$ related proteins (CFHRs). Mol Immunol. 2013 Dec $15 ; 56(3): 170-80$

9 Valoti E, Alberti M, Iatropoulos P, Piras R, Mele C, Breno M, et al. Rare functional variants in complement genes and anti-FH autoantibodies-associated aHUS. Front Immunol. 2019 May $1 ; 10: 853$. 